\title{
BIFURCATION AT EIGENVALUES OF ODD MULTIPLICITY
}

\author{
DAVID WESTREICH
}

\begin{abstract}
The most general form of the bifurcation problem is considered. Under reasonable hypotheses, "eigenvalues" of odd multiplicity are shown to be bifurcation points.
\end{abstract}

Introduction. The most general form of the bifurcation problem was considered by Crandall and Rabinowitz [2]. Using rather elegant methods, they solved the problem at simple eigenvalues and characterized the bifurcating solution branch. Their techniques are dependent on the simplicity of the eigenvalue and apparently cannot be extended to eigenvalues of higher multiplicity. Using techniques similar to those employed by the author in [6], we will show that, under slightly weaker hypotheses than those assumed by Crandall and Rabinowitz, eigenvalues of odd multiplicity are bifurcation points. The proof, while showing the existence of solutions, is nonconstructive and does not lead to a characterization of the solutions. The results obtained can be applied to the solution of the differential equations considered in [2], with the dimensions of the appropriate null spaces being odd instead of one.

To describe our work, we let $W$ and $Y$ be real Banach spaces and $G$ a continuous map of an open subset of $W$ into $Y$. Suppose there is a simple continuous nonclosed curve $w(t)$, for $t$ in an interval about zero, such that $G(w(t)) \equiv 0$. Following Crandall and Rabinowitz [2], we call $w(0)$ a bifurcation point for the equation $G(w)=0$ with respect to the curve, if every neighborhood of $w(0)$ contains zeros of $G$ not lying on $w(t)$. The basic concern of our paper is that of finding the bifurcation points for $G=0$ with respect to the given curve.

Main results. We consider first a more specialized situation where $W$ is of the form $R \times X, X$ is a real Banach space, $G$ is a nonlinear continuously (Fréchet) differentiable [5, p. 40] map of a neighborhood of $(0,0)$ into $Y$ and $G(t, 0)=0$ for all $t$ near zero.

Received by the editors February 5, 1973.

AMS (MOS) subject classifications (1970). Primary 58C99; Secondary 46B99.

Key words and phrases. Bifurcation, eigenvalues, odd multiplicity, Banach space, Fréchet differentiable, Fredholm operator, index zero, implicit function theorem, contraction map, continuous curve.

(c) American Mathematical Society 1973 
THEOREM A. Let $G$ be a continuously differentiable map of a neighborhood of $(0,0) \in \boldsymbol{R} \times X$ into $Y$ and suppose

(a) $G(t, 0)=0$ for all $t$ near 0 ,

(b) the partial derivative $G_{x}(t, 0)$ is a continuously differentiable function of $t$,

(c)

$$
\left\|G_{t}(t, x)-G_{t x}(t, 0) x\right\| \leqq k(t, x)\|x\|
$$

where $k(t, x) \rightarrow 0$ as $(t, x) \rightarrow(0,0)$,

(d) $G_{x}(0,0)$ is a Fredholm operator of index zero $[4, p$. 103] and $\operatorname{dim} N\left(G_{x}(0,0)\right)$ is odd, and

(e) $G_{t x}(0,0) x \notin R\left(G_{x}(0,0)\right), x \in N\left(G_{x}(0,0)\right)$ and $x \neq 0$.

Then $t=0$ is a bifurcation point of the equation

$$
G(t, x)=0 .
$$

Here $N()$ and $R($ ) denote the null space and range.

REMARK. It is readily verified that the existence and continuity of $G_{t x}(t, x)$ imply conditions (b) and (c) (see the proof of Theorem B).

Proof. Choose a complement $V$ of $N\left(G_{x}(0,0)\right)$ and let $S$ be the restriction of $G_{t x}(0,0)$ to $N\left(G_{x}(0,0)\right)$. Then by our hypotheses $S$ is invertible, $Y=$ $R(S) \oplus R\left(G_{x}(0,0)\right)$, and $G(t, x)=G^{* *}(t, x)+G^{*}(t, x)$ where $G^{* *}(t, x) \in$ $R(S)$ and $G^{*}(t, x) \in R\left(G_{x}(0,0)\right)$ [4]. Thus our problem is equivalent to that of finding solutions $(t, u, v) \in R \times N\left(G_{x}(0,0)\right) \times V$ of the system of equations

$$
\begin{aligned}
G^{* *}(t, u+v) & =0 \\
G^{*}(t, u+v) & =0 .
\end{aligned}
$$

Since $G_{v}^{*}(0,0)$ is a linear homeomorphism of $V$ onto $R\left(G_{x}(0,0)\right)$, an application of the implicit function theorem [3, p. 265] to equation (3) shows the existence of a uniquely determined function $f(t, u)=v$ such that $G^{*}(t, u+f(t, u)) \equiv 0$ for $(t, u)$ in a neighborhood of $(0,0)$. Moreover, $f$ is continuously differentiable. Hence it suffices to find solutions in $\boldsymbol{R} \times$ $N\left(G_{x}(0,0)\right)$ of the equation

$$
\begin{aligned}
G^{* *}(t, u+f(t, u)) & =H(t, u+f(t, u))+F(t)(u+f(t, u))+t S u \\
& =0
\end{aligned}
$$

where $F(t)+t S=G_{x}^{* *}(t, 0)$. Choose an inner product $\langle$,$\rangle which makes$ $R(S)$ a Hilbert space [4]. Then taking inner products with respect to $S u$ and dividing by $\|S u\|^{2}=\langle S u, S u\rangle$, we get from (4) the equation

$$
-\|S u\|^{-2}\langle H(t, u+f(t, u))+F(t)(u+f(t, u)), S u\rangle=t .
$$


Let $M(t, u)$ be the function defined by the left-hand side of $(5)$. We shall show that, for a suitably determined set, $M(t, u)$ is continuous and is a contraction map [3, p. 260] in $t$ for each fixed $u$.

To carry through the proof we let $N_{\delta}=\{(t, u)|| t \mid,\|u\| \leqq \delta\}$ and verify the following inequalities for each sufficiently small positive $\delta$ and $\left(t_{1}, u\right)$ and $\left(t_{2}, u\right) \in N_{\delta}$

(6) $\left\|H\left(t_{1}, u+f\left(t_{1}, u\right)\right)-H\left(t_{2}, u+f\left(t_{2}, u\right)\right)\right\| \leqq h_{1}(\delta)\|S u\|\left|t_{1}-t_{2}\right|$,

$$
\left\|F\left(t_{1}\right) f\left(t_{1}, u\right)-F\left(t_{2}\right) f\left(t_{2}, u\right)\right\| \leqq h_{2}(\delta)\|S u\|\left|t_{1}-t_{2}\right|,
$$

and

$$
\left\|F\left(t_{1}\right) u-F\left(t_{2}\right) u\right\| \leqq h_{3}(\delta)\|S u\|\left|t_{1}-t_{2}\right|,
$$

where $h_{i}(\delta) \rightarrow 0$ as $\delta \rightarrow 0$ for $i=1,2,3$.

Inequality (6) is established by first verifying that for $(t, u) \in N_{\delta}$

$$
\|f(t, u)\| \leqq k_{1}(\delta)\|u\|
$$

where $k_{1}(\delta) \rightarrow 0$ as $\delta \rightarrow 0$. For each sufficiently small $\delta$

$$
\|f(t, u)\|=\|f(t, u)-f(t, 0)\| \leqq\left\{\sup _{(t, u) \in N_{\delta}}\left\|f_{u}(t, u)\right\|\right\}\|u\|
$$

[3, p. 155]. By the implicit function theorem

$$
f_{u}(0,0)=-\left[G_{v}^{*}(0,0)\right]^{-1}\left[G_{u}^{*}(0,0)\right]=0 .
$$

Hence by continuity (9) follows from (10) and (11).

Next we show that for $(t, u) \in N_{\delta}$

$$
\left\|f_{t}(t, u)\right\| \leqq k_{2}(\delta)\|u\|
$$

where $k_{2}(\delta) \rightarrow 0$ as $\delta \rightarrow 0$. By the implicit function theorem, we find

$$
\begin{aligned}
\left\|f_{t}(t, u)\right\| \leqq & \left\|\left[G_{v}^{*}(t, u+f(t, u))\right]^{-1}\right\| \\
& \times\left\{\left\|G_{t}^{*}(t, u+f(t, u G))-{ }_{t x}^{*}(t, 0)(u+f(t, u))\right\|\right. \\
& \left.+\left\|G_{t x}^{*}(t, 0)(u+f(i, u))\right\|\right\}
\end{aligned}
$$

as $G_{t x}^{*}(0,0) u=0$ for $u \in N\left(G_{x}(0,0)\right)$, (12) follows from (1), (9) and (13).

Now we can verify (6). For $\delta$ sufficiently small

$$
\begin{aligned}
&\left\|H\left(t_{1}, u+f\left(t_{1}, u\right)\right)-H\left(t_{2}, u+f\left(t_{2}, u\right)\right)\right\| \\
& \leqq\left\{\sup _{(t, u) \in N_{\delta}}\left\|H_{t}(t, u+f(t, u))\right\|\right\}\left|t_{1}-t_{2}\right|
\end{aligned}
$$


and by the chain rule

$$
\begin{aligned}
\| H_{t}(t, u+ & f(t, u)) \| \\
& \leqq\left\|H_{1}(t, u+f(t, u))\right\|+\left\|H_{2}(t, u+f(t, u))\right\|\left\|f_{t}(t, u)\right\| .
\end{aligned}
$$

Thus, using the invertibility of $S$, (6) follows from (1), (12), (14) and (15).

To verify (7), we note

$$
\begin{aligned}
& \left\|F\left(t_{1}\right) f\left(t_{1}, u\right)-F\left(t_{2}\right) f\left(t_{2}, u\right)\right\| \\
& \leqq \\
& \leqq \\
& \leqq
\end{aligned}
$$

Inequality (7) thus follows from (16), (9) and (12).

Lastly, we verify (8).

$$
\left\|F\left(t_{1}\right) u-F\left(t_{2}\right) u\right\| \leqq\left\{\sup _{|t| \leqq \delta}\left\|F^{\prime}(t) u\right\|\right\}\left|t_{1}-t_{2}\right| .
$$

As $F^{\prime}(0) u=0$ for all $u \in N\left(G_{x}(0,0)\right)$, (8) follows from (17).

Next we show that for each small $\delta$, there is a $\delta_{1}$ such that

$$
|M(t, u)| \leqq \delta
$$

for all $|t| \leqq \delta_{1}$ and $0<\|u\| \leqq \delta_{1}$ and thus

Now

$$
\lim _{(t, u) \rightarrow(0,0) ; u \neq 0} M(t, u)=0 .
$$

$$
\begin{aligned}
& |M(t, u)| \\
& \qquad \begin{array}{l}
\leqq S u \|^{-1}\{\|H(t, u+f(t, u))-H(0, u+f(0, u))\| \\
\quad+\|F(t)\|[\|u\|+\|f(t, u)\|]+\|H(0, u+f(0, u))\|\} .
\end{array}
\end{aligned}
$$

As $H_{u+v}(0,0)=0$ it follows from the definition of the derivative that

$$
\|H(0, u+f(0, u))\|=o(\|u\|) .
$$

Hence, since $F(0)=0,(18)$ and (19) follow from (6), (9), (21) and (20). Choose a $\delta$, such that (6), (7), (8) and (18) are satisfied for $(t, u) \in A$,

$$
A=\{(t, u)|0<\|u\| \leqq \delta,| t \mid \leqq \delta\} \cup\{(0,0)\}
$$

and $h_{i}(\delta) \leqq \frac{1}{4}, i=1,2,3$. Then using (6), (7) and (8) one can easily show that for $\left(t_{1}, u\right),\left(t_{2}, u\right) \in A,\left|M\left(t_{1}, u\right)-M\left(t_{2}, u\right)\right| \leqq \frac{3}{4}\left|t_{1}-t_{2}\right|$. Hence we may apply the contraction mapping principle and for each $0<\|u\| \leqq \delta$ there 
exists a unique $t=t(u),|t| \leqq \delta$, such that $M(t, u)=t$. Moreover, if we set $t(0)=0$, then $t(u)$ is continuous for $\|u\| \leqq \delta$. Indeed, define $M_{0}(u)=$ $M(0, u)$ and for each integer $n \geqq 0$ let $M_{n+1}(u)=M\left(M_{n}(u), u\right)$. Each $M_{n}(u)$ is continuous for $\|u\| \leqq \delta$ and $M_{n}(0)=0$.

A straightforward induction shows $\left|M_{n}(u)-M_{n-1}(u)\right| \leqq\left(\frac{3}{4}\right)^{n}\left|M_{0}(u)\right| \leqq$ $\left(\frac{3}{4}\right)^{n}$. Hence for any integers $n \leqq m$

$$
\left|M_{m}(u)-M_{n}(u)\right| \leqq \sum_{i=n}^{m-1}\left|M_{i+1}(u)-M_{i}(u)\right| \leqq \sum_{i=n}^{\infty}\left(\frac{3}{4}\right)^{i}
$$

so that as $n, m \rightarrow \infty$ the above term tends to zero. We thus conclude $\left\{M_{n}(u)\right\}$ is a uniformly convergent sequence of continuous functions. The limit function $t(u)$ is therefore continuous and $t(0)=0$.

Now we are ready to show 0 is a bifurcation point of (2). To complete the proof we shall employ the following lemma. If $K$ is a continuous map of an $\boldsymbol{R}^{n}$ sphere, $S_{r}$ of radius $r$, into $\boldsymbol{R}^{n}$ and $n$ is odd, then there is an $x \in S_{r}$ and $a \lambda \in \boldsymbol{R}$ such that $K(x)=\lambda x[1, \mathrm{p} .51]$. This lemma will be used to solve the odd dimensional bifurcation problem $S^{-1} G^{* *}(t, u+f(t, u))=0$ with zero linearized part.

Let

$$
\hat{G}(t, u)=-S^{-1}[H(t, u+f(t, u))+F(t)(u+f(t, u))] .
$$

By the above lemma for each sufficiently small $r$ there is a $u_{r} \in N\left(G_{x}(0,0)\right)$ of norm $r$ and a $\lambda_{r} \in \boldsymbol{R}$ such that

$$
\hat{G}\left(t\left(u_{r}\right), u_{r}\right)=\lambda_{r} u_{r} \text {. }
$$

Multiplying (22) by $S$ and taking inner products we obtain $M\left(t\left(u_{r}\right), u_{r}\right)=\lambda_{r}$. However, by the definition of $t(u), t\left(u_{r}\right)=\lambda_{r}$. Hence for each small $r>0$ there is a solution $\left(t\left(u_{r}\right), u_{r}+f\left(t\left(u_{r}\right), u_{r}\right)\right)$ of equation (1) and by the continuity of $t(u)$ and $f(t, u)$ it follows that $t=0$ is a bifurcation point.

This result can be used to solve a more general bifurcation problem.

THEOREM B. Let $W, Y$ be Banach spaces, $K$ a twice continuously differentiable map of an open subset of $W$ into $Y$. Let $w(t)$ be a simple nonclosed continuously differentiable curve such that $K(w(t)) \equiv 0$ for $t$ in an interval about zero. Suppose

(a) $w^{\prime}(0) \neq 0$

(b) $\operatorname{dim} N\left(K^{\prime}(w(0))\right)=2 n, \operatorname{codim} R\left(K^{\prime}(w(0))\right)=2 n-1, n$ a positive integer,

(c) $N\left(K^{\prime}(w(0))\right)$ is spanned by $w^{\prime}(0)$ and $v_{1}, \cdots, v_{2 n-1}$, and

(d) $K^{\prime \prime}(w(0))\left(w^{\prime}(0), v\right) \notin R\left(K^{\prime}(w(0))\right), v \in \operatorname{span}\left\{v_{1}, \cdots, v_{2 n-1}\right\}, v \neq 0$.

Then $w(0)$ is a bifurcation point of $K(w)=0$ with respect to $w(t)$.

Proof. Arguing as in the beginning of $\S 1$ of [2] we see that proving our theorem is equivalent to showing $t=0$ is a bifurcation point of

$$
G(t, x)=K(w(t)+x)=0, \quad(t, x) \in \boldsymbol{R} \times X,
$$


where $X$ is a complement of span $\left\{w^{\prime}(0)\right\}$ in $W$. Moreover, $G_{t x}(t, x)$ exists and is continuous and $G(t, x)$ satisfies conditions (a), (b), (d) and (e) of Theorem A. Hence, we are done once we have shown $G(t, x)$ also satisfies (c). But this is immediate from the definition of the derivative of $G_{t}$ since $G(t, 0) \equiv 0$ implies $G_{t}(t, 0) \equiv 0$.

\section{REFERENCES}

1. M. S. Berger and M. S. Berger, Perspectives in nonlinearity. An introduction to nonlinear analysis, Benjamin, New York, 1968. MR 40 \#4971.

2. M. G. Crandall and P. H. Rabinowitz, Bifurcation from simple eigenvalues, J. Functional Analysis 8 (1971), 321-340. MR 44 \#5836.

3. J. Dieudonné, Foundations of modern analysis, Pure and Appl. Math., vol. 10, Academic Press, New York, 1960. MR 22 \#11074.

4. S. Goldberg, Unbounded linear operators. Theory and applications, McGraw-Hill, New York, 1966. MR 34 \#580.

5. M. M. Vainberg, Variational methods for the study of nonlinear operators, GITTL, Moscow, 1956; English transl., Holden-Day, San Francisco, Calif., 1964. MR 19, $567 ; 31$ \#638.

6. D. Westreich, Banach space bifurcation theory, Trans. Amer. Math. Soc. 171 (1972), 135-156.

Department of Mathematics, University of the Negev, Beer-Sheva, IsRael 